
retinal tubulation in Chinese patients after

SUBJECT AREAS:

RETINAL DISEASES

ANATOMY

Received

25 July 2014

Accepted

3 December 2014

Published

7 January 2015

Correspondence and requests for materials should be addressed to R.H. (woodshua@126. \title{
intravitreal injections of ranibizumab
}

\author{
Rui Hua, Limin Liu, Yuedong Hu \& Lei Chen
}

Department of Ophthalmology, First Hospital of China Medical University, Shenyang, China.

To investigate the occurrence and progression of outer retinal tubulation (ORT) in Chinese patients after intravitreal ranibizumab injections, using spectral domain optical coherence tomography (SD-OCT) with eye tracking function. 15 age related macular degeneration (AMD) and 6 polypoidal choroidal vasculopathy (PCV) eyes of 21 patients were enrolled and assessed by SD-OCT. One patient received photodynamic therapy (PDT) previously, and all patients received intravitreal injections of ranibizumab. At baseline, only one AMD eye (4.8\%) showed ORT, which appeared as round or ovoid hyporeflective spaces with hyperreflective borders. During the follow ups, ORT was identified in nine of 15 AMD eyes $(60.0 \%)$ and one of six PCV eyes $(16.7 \%)$. These new ORTs in 10 eyes were originated from the intraretinal fluid. Inner nuclear layer (INL), outer plexiform layer (OPL) and outer nuclear layer (ONL) were pulled down to form "cynapsis", separating each ORT. However, ORT in 3 eyes disappeared after intravitreal ranibizumab injections. This is the first observation on the occurrence and progression of ORT in Chinese AMD and PCV patients, in a point to point manner. The ORT could become stable or disappear after ranibizumab injections, and outer retina involved in the process of ORT formation.

R ecent investigations have demonstrated outer retinal tubulation (ORT) as minute retinal architectural change, in patients with various retinal disorders ${ }^{1}$, which are not detectable clinically. It is reported that ORT is a rearrangement of the photoreceptor layer in response to injury, in which surviving photoreceptors form new lateral connections with neighboring cells ${ }^{2}$. Recently, with the widespread adoption of using spectral domain optical coherence tomography (SD-OCT) in diagnosing and monitoring retinal diseases, ORT has become a more commonly recognized occurrence in eyes with focal disruptions of the outer retina related to multiple diagnoses ${ }^{1}$. For example, it has been observed in eyes with choroidal neovascularization (CNV) because of age-related macular degeneration (AMD), and in eyes with geographic atrophy, as well as retinal degenerations (retinitis pigmentosa, Stargardt disease, or pattern dystrophy) ${ }^{3}$.

On histopathologic sections, these rosette like structures were characterized by photoreceptor disruption ${ }^{4}$. Similarly, on SD-OCT profile, it appears as round or ovoid hyporeflective structures with hyperreflective margins, and a branching pattern of the tubes may be detected with curved en face SD-OCT ${ }^{5}$. In particular, the margins are believed to represent the ellipsoid portion of the photoreceptor inner segment ${ }^{6}$. In addition, the process of ORT formation was regarded as with outward folding of photoreceptors at the junction of intact and disrupted the ellipsoid portion of the photoreceptor inner segment initially and formation of a long, ovoid tubular complex eventually ${ }^{1}$.

Concurrently, there may be several points of attachment to neighboring neural elements with this deformed photoreceptor layer ${ }^{1}$. In this process, inner nuclear layer (INL), outer plexiform layer (OPL) and outer nuclear layer (ONL) are likely pulled down or collapse to appear numerous ramifications. We term this pattern as "cynapsis."

Because of the cystic-like appearance on OCT B-scans, ORT may be confused with cystoid macular edema (CME) related to leakage from CNV or other retinal diseases. On the contrary to CME, Zweifel SA, et al. report that, ORT always remained stable over time, even in patients receiving intravitreal anti-vascular endothelial growth factor (anti-VEGF) injections ${ }^{1}$. However, there have been no such long period studies investigating the occurrence and progression of ORT in a point to point manner in Chinese patients, after anti-VEGF injections. This is thus investigated in this study. 


\section{Results}

At baseline, only one AMD eye (4.8\%) showed ORT, which appeared as round or ovoid hyporeflective spaces with hyperreflective borders, overlying areas of retinal pigment epithelial (RPE) alteration and subretinal fibrosis (Figure 1). In addition, intraretinal cysts and subretinal fluid (SRF) were found in 17 out of 21 recruited eyes. During the follow ups, ORT was identified in nine of 15 AMD eyes $(60.0 \%)$ and one of six PCV eyes (16.7\%). From the point to point observations, these new ORTs in 10 eyes were originated from the intraretinal fluid that presumably damaged the outer retinal architecture, prior to treatment. In addition, INL, OPL and ONL were pulled down to form "cynapsis", separating each ORT (Figure 2). Moreover, ORT in 8 eyes remained stable during the follow up periods, but the other ORT disappeared gradually after intravitreal anti-VEGF injections.

Report of cases. Case 1. A 54-year-old man was diagnosed as longstanding neovascular AMD in his right eye. BCVA was 35 in ETDRS related to subretinal fluid, exudation and PRE elevation. After antiVEGF injections, INL, OPL and ONL showed arch like appearance, with disrupted and involute the ellipsoid portion of the photoreceptor inner segment, but without any SRF and retinal cysts. And then, the ORT was formed as multiple round and ovoid structures with hyperreflective borders visible. At the same time, INL, OPL and ONL were pulled down further and the intervals of disrupted the ellipsoid portion of the photoreceptor inner segment were enlarged. Next, INL, OPL and ONL were pulled down to form "cynapsis", separating each ORT. Finally, the ORT disappeared, with the confused structures of INL, OPL, and ONL left (Figure 3).

Case 2. A 78-year-old male was diagnosed as wet AMD in his left eye. The BCVA was 29 in ETDRS. At his first visit, OCT showed CNV, CME and RPE elevation. After anti-VEGF injections, the ORT was observed closed to the regions where there had been retinal cysts, and
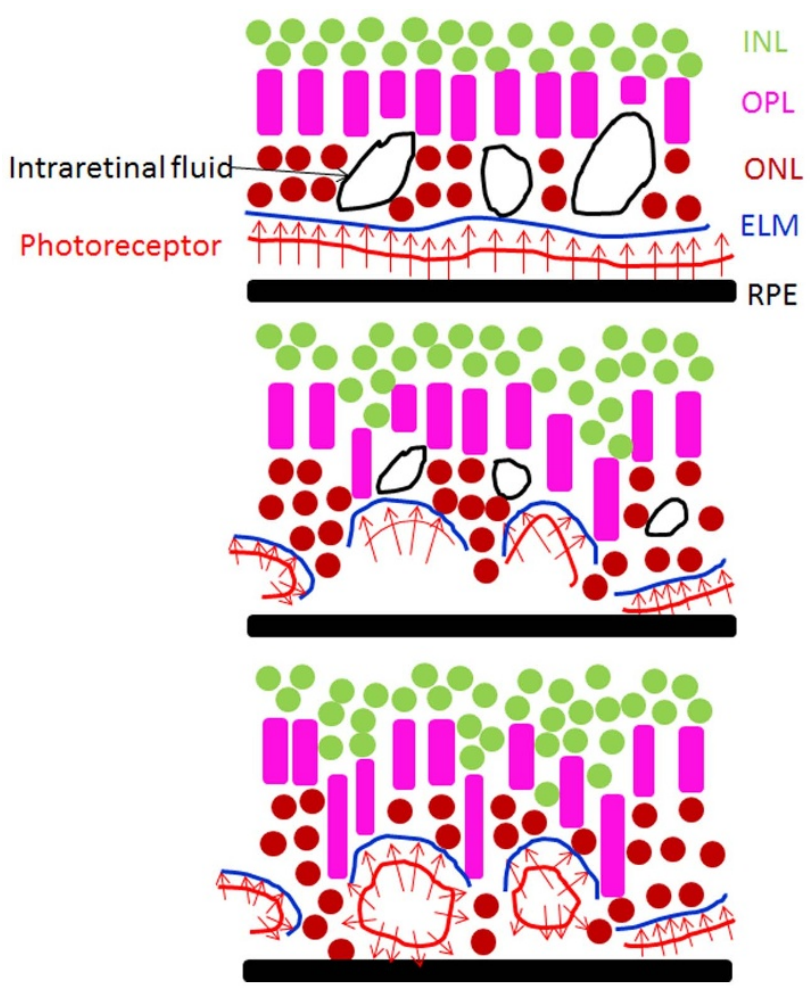

Figure $2 \mid$ The schema of the ORT formation. The new ORTs were usually originated from the intraretinal fluid, and INL, OPL and ONL were pulled down to form "cynapsis", separating each ORT.

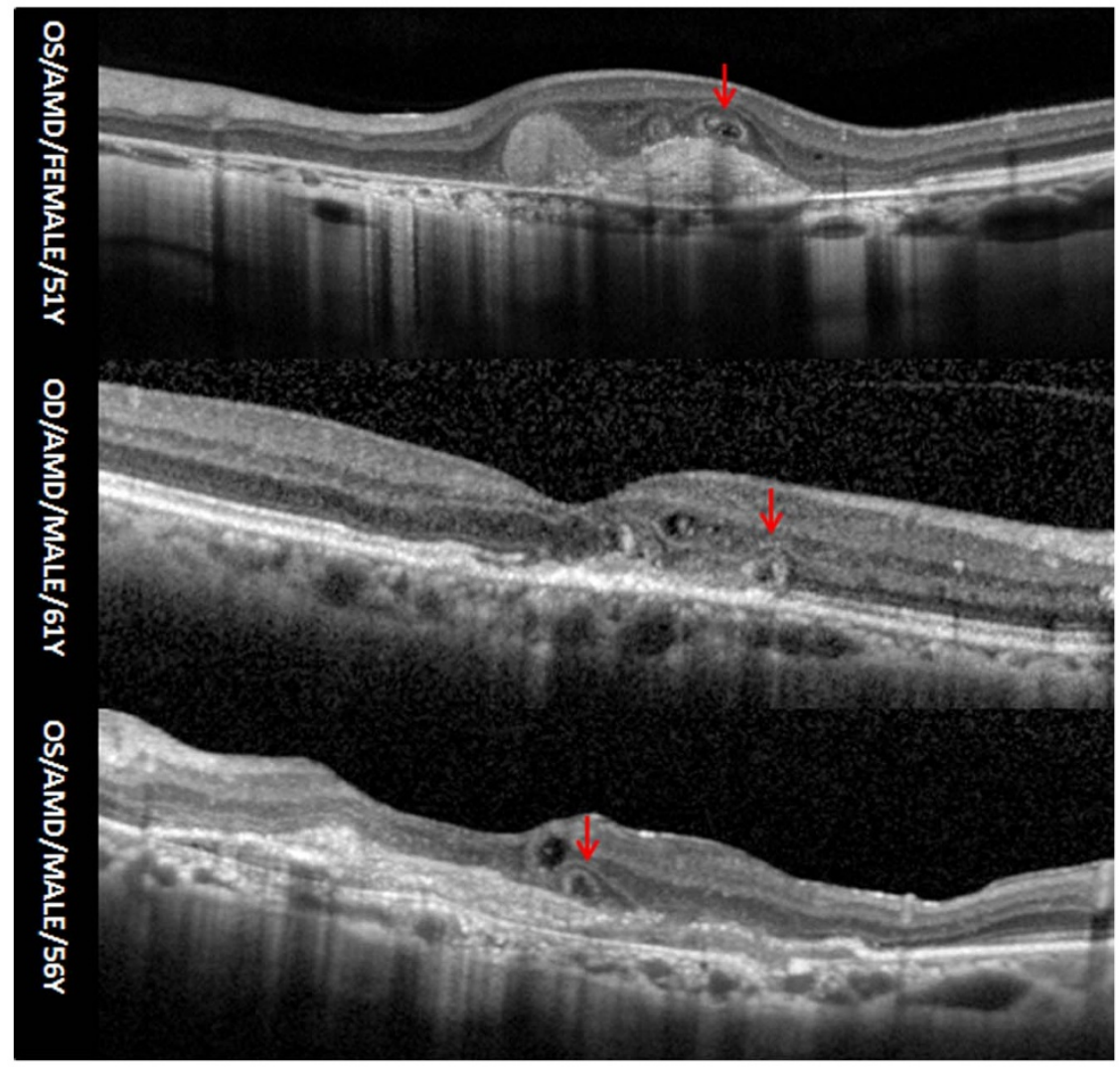

Figure $1 \mid$ The ORT on OCT profiles. 


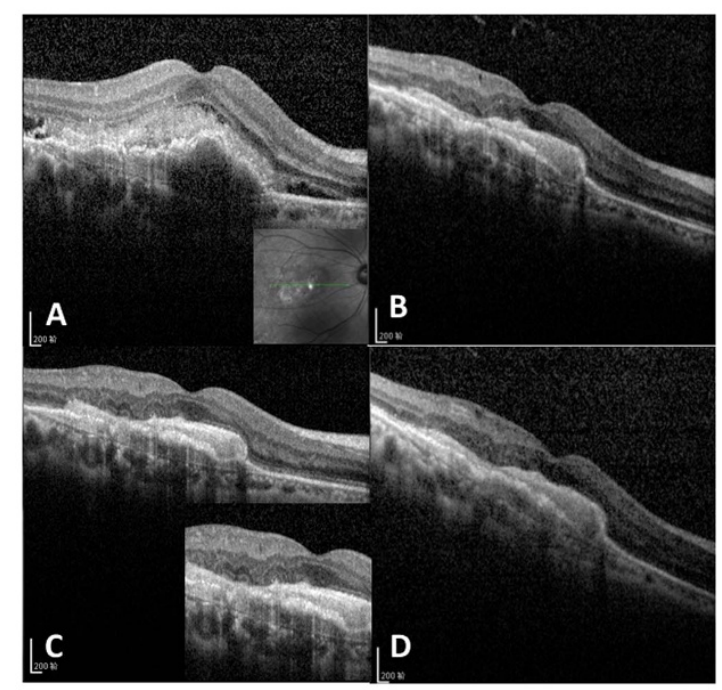

Figure 3 The SD-OCT images of case 1. Before injection, there were subretinal fluid, exudation and PRE elevation (A). B. one month after initial injection, INL, OPL and ONL showed arch like appearance and were pulled down, with disrupted and involute the ellipsoid portion of the photoreceptor inner segment, but without any SRF and retinal cysts. C. two months after initial injection, ORT was formed as multiple round and ovoid structures with hyperreflective borders visible. At the same time, INL, OPL and ONL were pulled down further to form "cynapsis", separating each ORT (insert image).D. one year after initial injection, the ORT disappeared, with the confused structures of INL, OPL, and ONL.

INL, OPL, as well as ONL were pulled down to form "cynapsis" near ORT. The retinal edema decreased. At the end of follow ups, the ORT collapsed (Figure 4).

Case 3. A 64-year-old male with neovascular AMD in his right eye was seen for a routine examination. The BCVA was 37 in ETDRS related to $\mathrm{CME}$, and $\mathrm{CNV}$. At baseline, the ORT was above the CNV. After intravitreal ranibizumab injections, the retinal cysts decreased, but the ORT remained stable (Figure 5).

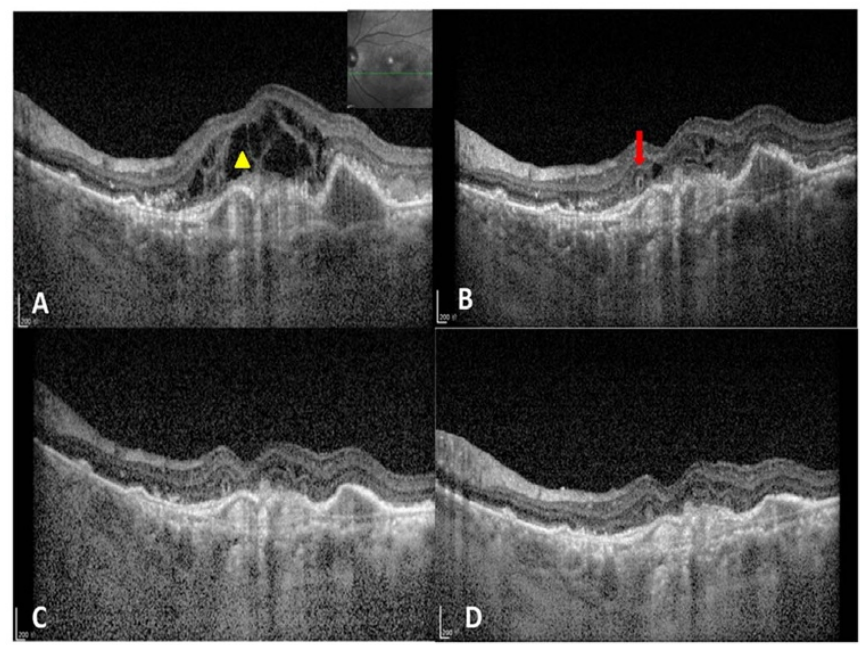

Figure $4 \mid$ The SD-OCT images of case 2.Before injection, OCT showed CNV, CME and RPE elevation. B. One month after initial injection, the ORT (red arrow) was observed closed to the regions where there had been retinal cysts (yellow triangle). C. three months after initial injection, INL, OPL and ONL were pulled down to form "cynapsis" near ORT. The retinal edema decreased. D. 13 months after initial injection, the ORT collapsed.

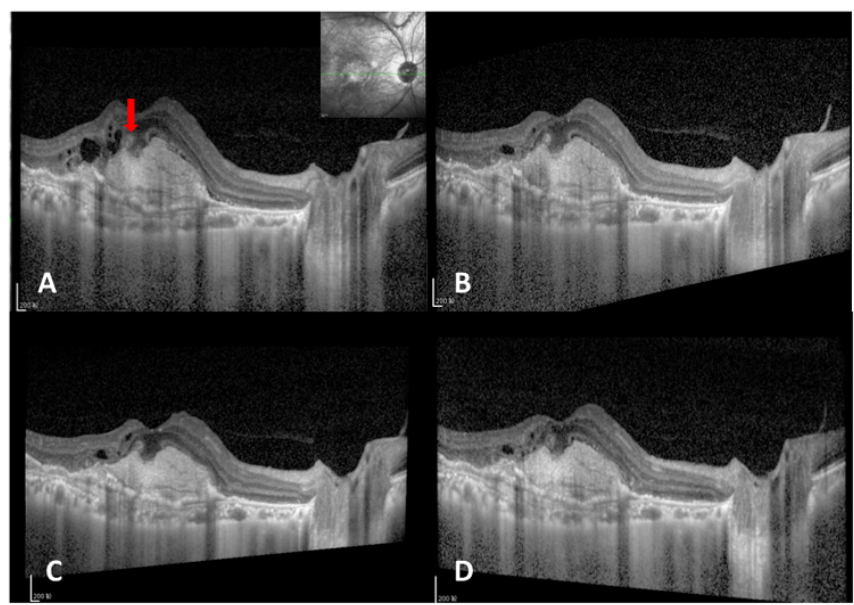

Figure $5 \mid$ The SD-OCT images of case 3. Initially, ORT was above the CNV (A.red arrow), together with intraretinal cysts and SRF. After injection, the retinal cysts decreased, but the ORT remained stable (B. one month, C. three months, D. eight months).

Case 4. A 65-year-old female was diagnosed as PCV in her left eye. The BCVA was 46 in ETDRS. Initially, there was a macular serous detachment, combined with RPE detachment and double layers sign on the OCT profile. One month after PDT, the previous macular serous detachment was absorbed partially, and ORT was formed besides the RPE detachment, and the lumina of ORT typically contain varying amounts of hyperreflective material. Additionally the INL, OPL and ONL were pulled down to form "cynapsis" near ORT. And then, the patient received intravitreal ranibizumab injections, and the ORT disappeared finally (Figure 6).

\section{Discussion}

Curcio, et al. firstly identified ORT in a histopathologic study of advanced AMD eye, and noted that surviving photoreceptors appeared to reorganize into interconnecting tubes over disciform scars ${ }^{7}$. Recent years, SD-OCT has revolutionized our ability to image the retina and analyze structural changes within the retina and choroid that develop with disease progression. So in the present study, using SD-OCT, we observed the occurrence of ORT in 10 eyes with AMD and PCV after anti-VEGF injection, and we noticed that the presence of ORT was higher in AMD cases. This process of ORT may be associated with the sublethal injury to the photoreceptors, loss of the interdigitation of the outer segments and RPE, disruption of tight junctions, reconstituting the ellipsoid portion of the photoreceptor inner segment junction, and forming a tubular structure eventually ${ }^{1}$. However, there was no such a report on the ORT in PCV cases, and we observed the ORT formed after PDT, and disappeared after antiVEGF injection in PCV. Additionally, the lumina of ORT in this PCV eye contained hyperreflective material. Because the exact mechanism of ORT is still unclear, the authors speculated the high occurrence of ORT in AMD might be due to the outer retina damage in AMD, compared with PCV.

Recently, Athanasios, et al. observed the ORT morphology in geographic atrophy using adaptive optics-optical coherence tomography, and found that the external limiting membrane, the ellipsoid portion of the photoreceptor inner segment, and RPE were loss surrounding the $\mathrm{ORT}^{8}$. Additionally, Goldberg NR, et al. also reported that the ORT structures appeared to arise from the gradual reorganization and invagination of the ellipsoid portion of the photoreceptor inner segment and, the external limiting membrane, appearing to form their hyperreflective Border ${ }^{3}$. As a supplementary, in the present study, we observed not only the occurrence and progression of ORT, but also the outer retina changes on the OCT 


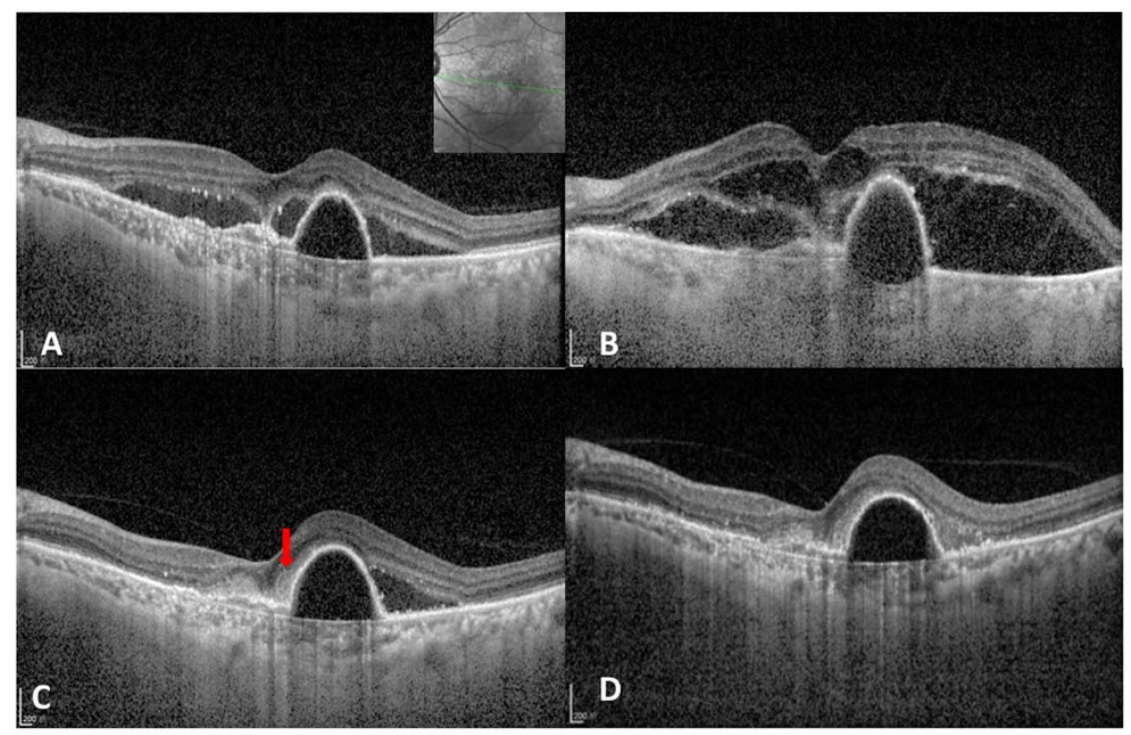

Figure 6 The SD-OCT images of case 4. Before injection, there was a macular serous detachment, combined with RPE detachment and double layers sign on the OCT profile. B. Three days after PDT, the sub-macular exudation increased and the RPE detachment was enlarged. C. one month after one session PDT, the previous macular serous detachment was absorbed partially, and ORT was formed besides the RPE detachment, and the lumina of ORT typically contain varying amounts of hyperreflective material, and INL, OPL and ONL were pulled down to form "cynapsis" near ORT. D. Four months after initial injection, the ORT disappeared.

profiles in AMD and PCV cases received anti-VEGF injections, firstly. The ORT was identified as circular or ovoid structures with hyperreflective borders, resembling the findings of CME and SRF, and some ORTs were originated from the substantial intraretinal fluid that presumably damaged the outer retinal architecture, prior to treatment. Similarly, this was in line with Freund's studies ${ }^{1,2}$. Before anti-VEGF injections, intraretinal and subretinal exudation damages the photoreceptor layer inducing focal loss of cells, disruption of tight junctions with neighboring cells, and loss of the interdigitation with the RPE. With resolution of fluid after treatment, the remaining cells reestablish lateral connections in the process of tubulation $^{1}$.

In addition, INL, OPL and ONL were pulled down to form "cynapsis", separating each ORT, which has not been reported previously. Moreover, unlike previous reports that in most of the cases, ORT remained stable over time, after anti-VEGF injections ${ }^{1}$, the ORT disappeared in 3 eyes after injections in this study. One possible explanation may be that there was a communicating structure between subretinal space and ORT, and the anti-VEGF treatment could then transiently collapse the ORT structures by reducing the leakage of fluid into the tubular network ${ }^{1}$. And Goldberg NR, et al. reported that both gain and loss of ORT were observed in some cases using the eye-tracking feature of SD-OCT and close analysis of serial sections in the OCT image sets, during follow-up ${ }^{3}$. However, we do not know the exact reasons for disappeared ORT and the confused structures of INL, OPL, and ONL. More researches are needed in this aspect.

Most commonly, ORT is observed in eyes with CNV in AMD, and usually is confused with CME related to leakage from CNV. Because ORT reflects common damage to the outer retina and/or RPE, and it may be an indicator of underlying disease stage and severity ${ }^{3}$, the understanding of ORT (both its occurrence and progression) is very essential for our clinical practice.

In conclusion, to the best of our knowledge, this is the first report on the occurrence and progression of ORT after intravitreal injections of ranibizumab from China. The point to point follow up technique was employed to trace the morphological changes of ORT. The authors suggested that the ORT might become stable or disappear after anti-VEGF injection, and except for the ellipsoid portion of the photoreceptor inner segment and RPE, the INL, OPL and ONL also involved in the process of ORT formation. In addition, ORT could be found in not only AMD, but also PCV, after PDT, which might be as a supplementary for previous studies.

There were some limitations in this study. For example, the small samples in the research and we did not observe the angiographic characteristics of ORT. More studies are needed.

\section{Methods}

Patients. This retrospective study involved the analysis of 21 eyes from 21 patients, enrolled in Department of Ophthalmology Outpatient, First Hospital of China Medical University, from Sep 2012 to Jul 2014, including 12 males and 9 females, with a mean age of 62.5 (range 51-78) years old. One patient received photodynamic therapy (PDT) previously. The diagnoses of 15 AMD and 6 polypoidal choroidal vasculopathy (PCV) were made by BERN reading center (Switzerland) partially, according to the stereo fundus photograph, SD-OCT, fluorescein angiography and indocyanine green angiography results. The inclusion criteria were (1) older than 50 years old; (2) best-corrected visual acuity (BCVA) was between 78-23 in ETDRS. Besides, the exclusion criteria were the patients with pregnant, stroke, myocardial infarction, hypertension, or with eye diseases, such as cataract, severe vitreous hemorrhage, rhegmatogenous retinal detachment, proliferative diabetic retinopathy, macular hole, idiopathic CNV, central serous chorioretinopathy, ocular histoplasmosis, pathologic myopia, and retinal scar within 0.5 optic disc. In addition, the patients received anti-VEGF therapy, previously, will also been excluded. The mean BCVA of the 21 eyes was 51 in ETDRS (35-64), and the mean central foveal thickness was $440 \mu \mathrm{m}(322-675)$ at baseline. All research and measurements adhered to the tenets of the Declaration of Helsinki, and this study was approved through the Medical Research Ethics Committee of First Hospital of China Medical University. Written informed consent was obtained from all participants.

Imaging Acquisition. SD-OCT (Spectralis HRA+OCT; Heidelberg Engineering, Heidelberg, Germany) with automatic real-time (ART) tracking was used in this study to achieve data acquisition rates of up to 40,000 axial scans/second. Volume scan with 49 lines $\left(20^{\circ} \times 20^{\circ}\right)$ and 9 frames in ART were performed in the macula. Single lines $\left(30^{\circ}\right)$ with 36 frames in ART were also performed, and centered at fovea. After diagnosis, the VEGF specific antibody ranibizumab (lucentis) $(0.5 \mathrm{mg} /$ $0.05 \mathrm{~mL}$ ) was injected intravitreally. The average number of injections were 7 (range 3-12). Point to point SD-OCT follow ups were performed at each visit, using eye tracking function in the Heidelberg eye explorer software (Figure 7). The follow up periods were during 8-15 months.

Two professional raters were responsible for the OCT images interpretation and ORT identification, according to Zweifel SA's study ${ }^{1}$. Discrepancies were referred to a fundus specialist (L. C.) for final determination.

Statistical analysis. All statistical analyses were performed using SPSS version 19.0 (Inc., Chicago, IL). Data are presented as the median (Min-Max). The OCT pattern 

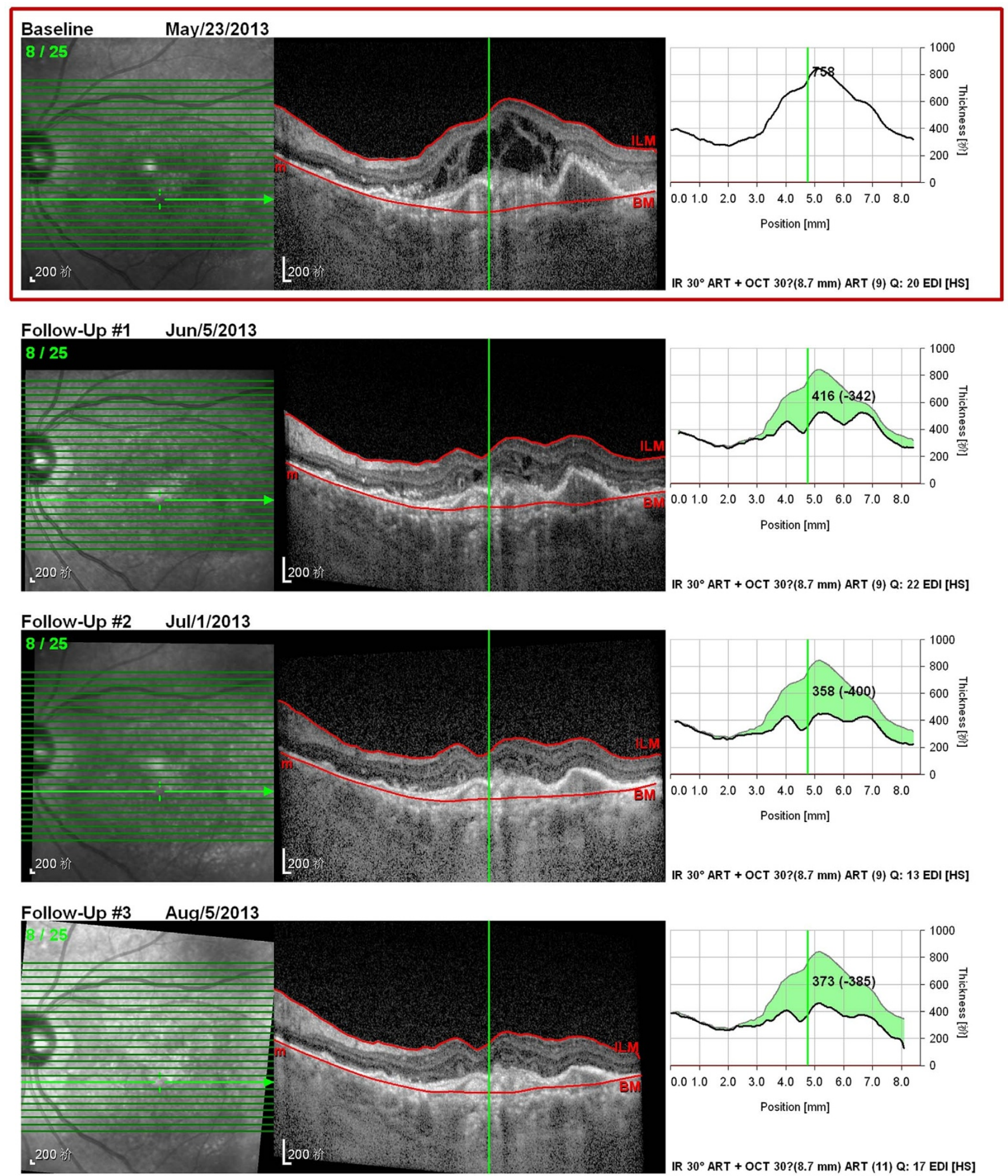

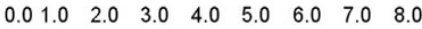

Position [mm]

IR $30^{\circ} \mathrm{ART}+\mathrm{OCT} 30 ?(8.7 \mathrm{~mm}) \mathrm{ART}$ (11) Q: $17 \mathrm{EDI}[\mathrm{HS}]$

Figure $7 \mid$ Point to point follow up report of SD-OCT in a 57-year-old female AMD patient (left eye).

and morphological changes of ORT were analyzed by descriptive statistics. Qualitative data were presented as frequency distributions.

1. Zweifel, S. A. et al. Outer retinal tubulation: a novel optical coherence tomography finding. Arch Ophthalmol. 127, 1596-1602 (2009).

2. Jung, J. J. \& Freund, K. B. Long-term follow-up of outer retinal tubulation documented by eye-tracked and en face spectral-domain optical coherence tomography. Arch Ophthalmol. 130, 1618-1619 (2012).
3. Goldberg, N. R., Greenberg, J. P., Laud, K., Tsang, S. \& Freund, K. B. Outer retinal tubulation in degenerative retinal disorders. Retina. 33, 1871-1876 (2013).

4. Tulvatana, W., Adamian, M., Berson, E. L. \& Dryja, T. P. Photoreceptor rosettes in autosomal dominant retinitis pigmentosa with reduced penetrance. Arch Ophthalmol. 117, 399-402 (1999).

5. EWolff, B., Matet, A., Vasseur, V., Sahel, J. A. \& Mauget-Faÿsse, M. En Face OCT Imaging for the Diagnosis of Outer Retinal Tubulations in Age-Related Macular Degeneration. J Ophthalmol. 2012, 542417 (2012). 
6. Spaide, R. F. \& Curcio, C. A. Anatomical correlates to the bands seen in the outer retina by optical coherence tomography: literature review and model. Retina. 31, 1609-1619 (2011)

7. Curcio, C. A., Medeiros, N. E. \& Millican, C. L. Photoreceptor loss in age-related macular degeneration. Invest Ophthalmol Vis Sci. 37, 1236-1249 (1996).

8. Panorgias, A. et al. Multimodal assessment of microscopic morphology and retinal function in patients with geographic atrophy. Invest Ophthalmol Vis Sci. 54, 4372-4384 (2013).

\section{Acknowledgments}

We thank the Dragon study of Novartis (CRFB002A2413 -- Dragon) in the management of partial patients, including the diagnoses, treatment, and follow-up.

\section{Author contributions}

Study concept and design: L.C. and R.H. Acquisition of data: R.H. L.M.L. and Y.D.H. Analysis and interpretation of data: R.H. and L.M.L. Drafting of the manuscript: R.H. Critical revision of the manuscript for important intellectual content: R.H. and L.C. All authors read and approved the final manuscript.

\section{Additional information}

Funding: This study was supported by the Liaoning Science and Technology Project (Project \#: 2011225014). The funders had no role in study design, data collection and analysis, decision to publish, and preparation of the manuscript.

Competing financial interests: The authors declare no competing financial interests.

How to cite this article: Hua, R., Liu, L., Hu, Y. \& Chen, L. The occurrence and progression of outer retinal tubulation in Chinese patients after intravitreal injections of ranibizumab. Sci. Rep. 5, 7661; DOI:10.1038/srep07661 (2015).

This work is licensed under a Creative Commons Attribution-NonCommercialNoDerivs 4.0 International License. The images or other third party material in this article are included in the article's Creative Commons license, unless indicated otherwise in the credit line; if the material is not included under the Creative Commons license, users will need to obtain permission from the license holder in order to reproduce the material. To view a copy of this license, visit http:// creativecommons.org/licenses/by-nc-nd/4.0/ 